\title{
ON THE CONTACT BETWEEN COMPLEX MANIFOLDS AND REAL HYPERSURFACES IN $C^{3}$
}

\author{
BY
}

THOMAS BLOOM ${ }^{1}$

\begin{abstract}
Let $m$ be a real $e^{\infty}$ hypersurface of an open subset of $C^{3}$ and let $p \in M$. Let $a^{1}(M, p)$ denote the maximal order of contact of a one-dimensional complex submanifold of a neighborhood of $p$ in $C^{3}$ with $M$ at $p$. Let $c^{1}(M, p)$ denote the $\sup \{m \in \mathbf{Z} \mid$ for all tangential holomorphic vector fields $L$ with $L(p) \neq$ 0 then $\left.L^{i_{0}} \bar{L}^{j_{0}} \ldots L^{i_{n}} \bar{L}^{j_{n}}\left(\mathcal{L}_{M}(L)\right)(p)=0\right\}$ where $i_{0}, \ldots, i_{n} ; j_{0}, \ldots, j_{n}$ are positive integers such that $\sum_{t=0}^{n} i_{t}+j_{t}=m-3$ and $\mathcal{L}_{M}(L)$ denotes the Levi form of $M$ evaluated on the vector field $L$.

THEOREM. If $M$ is pseudoconvex near $p \in M$ then $a^{1}(M, p)=c^{1}(M, p)$.
\end{abstract}

Introduction. The notion of type of a point on a real hypersurface in $\mathbf{C}^{2}$ was introduced by J. J. Kohn [12]. In [1] this notion was generalized to $\mathbf{C}^{\mathbf{n}}$. The type of a point $p$ on a real hypersurface $M$ is an integer $\geqslant 2$ or $+\infty$. It may be characterized geometrically as the maximal order of contact of a nonsingular complex hypersurface with the real hypersurface $M$ at $p$. It may be characterized algebraically as the minimal length of commutator of the tangential holomorphic vector fields and their conjugates needed to obtain the "missing direction".

For domains $D$ in $\mathbf{C}^{2}$ the type of a boundary point is an important invariant. For $D$ pseudoconvex with smooth boundary $b D$, Kohn showed [12] that if $p \in b D$ is of type $m$ there is a local subelliptic estimate in the $\bar{\partial}$-Neumann problem of order $1 / m$. P. Greiner showed that this is the best possible estimate [11] (see also [16], [18]).

Recent progress [8], [15] has established subelliptic estimates on pseudoconvex domains in $\mathbf{C}^{n}$ with smooth real-analytic boundaries. The type of a boundary point gives the best possible subelliptic estimate for $(p, n-1)$ forms. However it is clear that in $\mathbf{C}^{n}(n>2)$ other invariants will be needed.

The work of Diederich-Fornaess and Kohn suggests that the order of contact of (possibly singular) subvarieties with $b D$ at a point $p \in b D$ is an important invariant. (The best possible order of subelliptic estimates in the $\bar{\partial}$-Neumann problem or even sufficient conditions in the case of $e^{\infty}$ boundaries are currently open problems [5].)

In this paper we consider a real pseudoconvex hypersurface $M$ in $C^{3}$ and the order of contact with one-dimensional complex submanifolds with $M$ at $p \in M$.

Received by the editors November 30, 1979 and, in revised form, February 15, 1980.

1980 Mathematics Subject Classification. Primary 32F20, 32F25.

Key words and phrases. $\bar{\partial}$-Neumann problem, pseudoconvex hypersurface, tangential holomorphic vector field, type of a point.

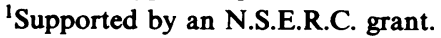


We give an algebraic characterization of the maximal order of contact (Theorem 2.14) in terms of properties of tangential holomorphic vector fields.

We state a conjecture for $M \subset \mathbf{C}^{n}$ and the orders of contact of complex submanifolds of any dimension. The main difficulty in proving this conjecture is the generalization of Theorem 4.2. This theorem gives, in fact, a stronger version of Theorem 2.14 in the special case that $M$ is weighted homogeneous.

The methods used in this paper are somewhat different from those of [1] or [3] which were almost entirely algebraic. Here we use results of Freeman and Nagano [9] and Diederich-Fornaess [8] which are less algebraic.

Also Theorem 2.14 (in contrast to the results of [1] or [2]) does depend on the pseudoconvexity of $M$. The example of 5.9 shows that Theorem 2.14 is not valid in general.

The paper is organized as follows: $\$ 1$ contains notation and definitions. $\$ 2$ contains the statement of the main result (Theorem 2.14) and related conjectures (2.16). $\$ 3$ reviews the notion of weighted holomorphic coordinates. The idea of weighted coordinates (see also [2], [10] and [18]) is used extensively. $\$ 4$ contains a special case of Theorem 2.14 (Theorem 4.2). $\$ 5$ reduces the general case of a pseudoconvex hypersurface in $\mathbf{C}^{3}$ to the special case handled in Theorem 4.2.

\section{Notation and definitions.}

1.1. Let $Z$ be a real $\bigodot^{\infty}$ submanifold of an open subset $U \subset \mathbf{C}^{n}$. Let $p \in Z$.

We denote by $T(Z, p)$ the real tangent space to $Z$ at $p$ and by $\operatorname{CT}(Z, p)$ its complexification. We denote by $T^{1,0}(Z, p)$ the subspace of $\mathrm{C} T(Z, p)$ consisting of holomorphic tangent vectors and by $T^{0,1}(Z, p)$ the subspace of conjugate holomorphic vectors.

We let $T^{h}(Z, p)=\left\{\operatorname{Re}(T) \mid T \in T^{1,0}(Z, p)\right\} . T^{h}(Z, p)$ is the maximal complex subspace of $T(Z, p)$ with respect to the canonical almost complex structure of $\mathbf{C}^{n}$. $T^{h}(Z, p)$ and $T^{1,0}(Z, p)$ are canonically isomorphic.

1.2. Let $M$ be a real $\bigodot^{\infty}$ hypersurface of an open set $U \subset \mathbf{C}^{n}$. Let $p \in M$. Then $T^{h}(M, p)$ is of (real) codimension one in $T(M, p)$ and $T^{1,0}(M, p) \oplus T^{0,1}(M, p)$ is of (complex) codimension one in $\mathbf{C T}(M, p)$.

$\cup_{q \in M} T^{1,0}(M, q)$ is a complex bundle which we denote by $T^{1,0}(M)$. Analogously, we have the bundles $T^{0,1}(M), T(M), C T(M)$ and $T^{h}(M)$.

We consider $M$ to be a portion of the boundary of an open set $G$ in $\mathbf{C}^{n}$. If $B$ is a small ball centered at $p$ then $B-M \cap B$ has two connected components, one of which is $G \cap B$. By a local defining function $\rho$ for $M$ near $p$ we mean a real $\bigodot^{\infty}$ function defined on an open ball $B$ centered at $p$ such that

$$
\begin{aligned}
M \cap B & =\{z \in B \mid \rho(z)=0\}, \\
d \rho & \neq 0 \text { on } M \cap B, \\
G \cap B & =\{z \in B \mid \rho(z)<0\} .
\end{aligned}
$$

We may choose local holomorphic coordinates $\left(z_{1}, \ldots, z_{n-1}, w\right)$ centered at $p$ such that $\rho$ has the form

$$
\rho=2 \operatorname{Re}(w)+R(z, w)
$$

where $R$ vanishes to order $\geqslant 2$ at $p$. 
1.3. Definitions. A holomorphic vector field on an open subset $U \subset \mathbf{C}^{n}$ is a vector field $F$ which can be written in the form

$$
F=\sum_{j=1}^{n} a_{j} \frac{\partial}{\partial z_{j}} \quad \text { where } a_{j} \in e^{\infty}(U) .
$$

The conjugate vector field is denoted by $\bar{F}$.

$$
\bar{F}=\sum_{j=1}^{n} \bar{a}_{j} \frac{\partial}{\partial \bar{z}_{j}} .
$$

Let $Z$ be a real $e^{\infty}$ submanifold of $U . F$ is a tangential holomorphic vector field to $Z$ if $F(q) \in \mathbf{C} T(Z, q)$ for all $q \in Z$ and $F(q) \in T^{1,0}\left(\mathbf{C}^{n}, q\right)$ for all $q \in U$.

1.4. Let $M$ be a real $\bigodot^{\infty}$ hypersurface of a neighborhood of a point $p \in \mathbf{C}^{n}$, and let $\rho$ be a defining function for $M$ near $p$. Then the holomorphic vector field $F$ is tangential to $M$ if, for all $q \in M$,

$$
\sum_{j=1}^{n} a_{j}(q) \frac{\partial \rho}{\partial z_{j}}(q)=0
$$

where $F$ is given in the form (1.3.1).

If one chooses local holomorphic coordinates such that $\rho$ has the form (1.2.4) then the tangential holomorphic vector fields given by

$$
L_{i}=\left(\partial \rho / \partial z_{i}\right)(\partial / \partial w)-(\partial \rho / \partial w)\left(\partial / \partial z_{i}\right) \text { for } i=1, \ldots, n-1,
$$

span $T^{1,0}(M, q)$ for all points $q$ near $p$.

1.5. The Levi form of $M$ will be denoted $\mathcal{L}_{M}(q, T)$ where $q \in M$ and $T \in$ $T^{1,0}(M, q)$. If $L$ is a tangential holomorphic vector field to $M$ then

$$
\mathcal{L}_{M}(q, L(q))=\langle[L, \bar{L}], \partial \rho\rangle(q)
$$

where [, ] denotes the commutator of vector fields and the brackets $\langle$,$\rangle denote$ the pairing between vectors and covectors.

Following Diederich and Fornaess [8] we will consider the Levi form on vectors in $T^{h}(M, q)$. Let $H \in T^{h}(M, q)$. There is a unique $T \in T^{1,0}(M, q)$ such that $\operatorname{Re}(T)=H$. Then one sets $\mathfrak{L}_{M}(q, H)=\mathfrak{E}_{M}(q, T)$.

Let $Z$ denote a real $\bigodot^{\infty}$ submanifold of $M$. Then [8] one defines

$$
T^{N}(Z, q)=\left\{H \in T^{h}(M, q) \cap T(Z, q) \mid \mathfrak{L}_{M}(q, H)=0\right\}
$$

and $T^{N}(Z)=\cup_{q \in Z} T^{N}(Z, q)$.

\section{The main result.}

2.1. Let $M$ be a real $e^{\infty}$ hypersurface of an open set $U \subset \mathbf{C}^{n}$ and let $p \in M$. In this section we define various numerical invariants attached to the germ of $M$ at $p$. There are three sets of invariants: $a^{s}(M, p)$ (Definition 2.3 ); $t^{s}(M, p)$ (Definition 2.6); and $c^{s}(M, p)$ (Definition 2.10). Each is defined for $s$ an integer, $1 \leqslant s \leqslant n-1$. Each invariant is an integer $\geqslant 2$ or $+\infty$.

The invariants $a^{s}(M, p)$ are geometric in nature and measure the maximal order of contact of $s$-dimensional complex submanifolds with $M$ at $p$. The other sets of invariants are algebraic in nature and depend on properties of tangential holomorphic vector fields. 
We conjecture (see Conjecture 2.16) relations between these invariants. This conjecture has been proven in the case $s=n-1$ in [1], [11] (see Theorems 2.12 and 2.13). That is, the equivalence between algebraic and geometric characterizations of the maximal contact between complex (nonsingular) hypersurfaces and real hypersurfaces has been established.

In this paper we prove (Theorem 2.14) that $a^{1}(M, p)=c^{1}(M, p)$ for $M$ pseudoconvex in $\mathbf{C}^{3}$. This result establishes equivalence between algebraic and geometric characterizations of the maximal contact between lower-dimensional complex submanifolds and real hypersurfaces (see also [6]).

The proofs of Theorems 2.12 and 2.13 are almost entirely algebraic. The proof of Theorem 2.14 is less so and relies on work of Diederich and Fornaess [8] and Freeman and Nagano [9].

2.2. Definition. Let $\boldsymbol{M}$ be a real $\bigodot^{\infty}$ hypersurface of an open set $U \subset \mathbf{C}^{n}$ and $p \in M$. Let $\rho$ be a defining function for $M$ near $p$. Let $X$ be a complex submanifold of a neighborhood of $p$ with $p \in X$. We define $X$ to have contact of order $r$ with $M$ at $p$ if the restriction of $\rho$ to $X, \rho \mid X$ vanishes to order $r$ at $p$.

The order of contact is independent of the choice of defining function for $\boldsymbol{M}$.

2.3. Definition. For $s$ an integer, $1 \leqslant s \leqslant n-1$, we set

$$
\begin{aligned}
& a^{s}(M, p)=\sup \{r \mid \text { there exists an } s \text {-dimensional complex } \\
& \text { submanifold } X \text { with contact of order } r \text { with } M \text { at } p\} .
\end{aligned}
$$

2.4. Let $F_{1}, \ldots, F_{\mu}$ be $\mu$ vector fields (or more generally, noncommuting indeterminates). Then the iterated commutator $\left[F_{\mu},\left[F_{\mu-1},\left[\cdots\left[F_{2}, F_{1}\right]\right]\right]\right]$ is said to be of length $\mu$.

Let $B$ be an $s$-dimensional (complex) subbundle of $T^{1,0}(M)$. We let $\mathfrak{T}_{1}(B)$ denote the module (over $C^{\infty}(U)$ ) spanned by tangential holomorphic vector fields $L$ such that $L(q) \in B(q)$ for all $q \in M$ together with the conjugates of such vector fields. For $\mu$ an integer $\geqslant 1$ we let $\mathfrak{T}_{\mu}(B)$ denote the module (over $\bigodot^{\infty}(U)$ ) spanned by commutators of length $\leqslant \mu$ of vector fields in $\Re_{1}(B)$.

2.5. Definition. We define the integral invariant $t(B, p)$ as follows.

$$
t(B, p)=m \text { if }\langle F, \partial \rho\rangle(p)=0 \text { for all } F \in \Re_{m-1}(B)
$$

but,

$$
\langle G, \partial \rho\rangle(p) \neq 0 \text { for some } G \in \mathfrak{N}_{m}(B) .
$$

2.6. Definition. We define the integral invariants $t^{s}(M, p)$ (for $s$ an integer $1 \leqslant s \leqslant n-1)$ as follows.

$$
t^{s}(M, p)=\sup \left\{t(B, p) \mid B \text { is an } s \text {-dimensional subbundle of } T^{1,0}(M)\right\} .
$$

2.7. Remark. $t^{n-1}(M, p)$ is the type of the point $p \in M$ as defined in [1].

2.8. Let $B$ be an $s$-dimensional complex subbundle of $T^{1,0}(M)$. We denote by $\operatorname{tr}_{B} E_{M}$ the trace of the Levi form of $M$ restricted to $B$.

2.9. Definition. We define the integral invariant $c(B, p)$ as follows. $c(B, p)=m$ if for any $m-3$ vector fields $F_{1}, \ldots, F_{m-3}$ of $\Re_{1}(B)$ we have $F_{1} \cdots F_{m-3}\left(\operatorname{tr}_{B} \varrho_{M}\right)(p)=0$, but for some choice of $m-2$ vector fields $G_{1}, \ldots, G_{m-2}$ of $\mathfrak{R}_{1}(B)$ we have $G_{1} \cdots G_{m-2}\left(\operatorname{tr}_{B} \mathcal{L}_{M}\right)(p) \neq 0$. 
2.10. Definition. We define the integral invariant $c^{s}(M, p)$ (for $s$ an integer $1 \leqslant s \leqslant n-1)$ as follows.

$$
c^{s}(M, p)=\sup \left\{c(B, p) \mid B \text { is an } s \text {-dimensional subbundle of } T^{1,0}(M)\right\} .
$$

2.11. Remark. The invariants $t(B, p), c(B, p), t^{s}(M, p)$ and $c^{s}(M, p)$ are all independent of the choice of defining function $\rho$ for $M$.

2.12. TheOREM [1, TheOREM 2.4]. $t^{n-1}(M, p)=a^{n-1}(M, p)$.

2.13. TheOREM [3, AdDENDUM]. If $M$ is pseudoconvex near $p$ then $c^{n-1}(M, p)=$ $a^{n-1}(M, p)$.

2.14. THEOREM. Let $M$ be a real $e^{\infty}$ hypersurface in an open set $U \subset \mathbf{C}^{3}$ and let $p \in M$. Suppose $M$ is pseudoconvex near $p$. Then $c^{1}(M, p)=a^{1}(M, p)$.

This is the main result of this paper. The proof is carried out in $\S \S 4$ and 5 .

2.15. We will give a slightly different formulation of the invariant $c^{1}(M, p)$ (Definition 2.10).

Let $L$ be a tangential holomorphic vector field with $L(p) \neq 0$. We define the integral invariant $c(L, p)$ as follows. $c(L, p)=m$ if for any choice of positive integers $k, i_{0}, \ldots, i_{k}, j_{0}, \ldots, j_{k}$, with $\sum_{t=0}^{k}\left(i_{t}+j_{t}\right)=m-3$, we have

$$
L^{i_{0}} \bar{L}^{j_{0}} \cdots L^{i_{k}} \bar{L}^{j_{k}}\langle[L, \bar{L}], \partial \rho\rangle(p)=0,
$$

whereas for some choice of positive integers $k, i_{0}, \ldots, i_{k}, j_{0}, \ldots, j_{k}$, with

$$
\sum_{t=0}^{k}\left(i_{t}+j_{t}\right)=m-2,
$$

then the expression on the left side of $(2.15 .1)$ is $\neq 0$.

It is easy to show that $c^{1}(M, p)=\sup \{c(L, p) \mid L$ is a tangential holomorphic vector field to $M$ and $L(p) \neq 0\}$.

It is the above formulation of the invariant $c^{1}(M, p)$ which we will use in the proof of Theorem 2.14.

We also note that if $f$ is a $e^{\infty}$ function such that $f(p) \neq 0$ then $c(L, p)=$ $c(f L, p)$.

2.16. Conjecture. Let $M$ be a real $\bigodot^{\infty}$ hypersurface in an open set $U \subset \mathbf{C}^{n}$ and let $p \in M$. Suppose $M$ is pseudoconvex near $p$. Then for $1<s \leqslant n-2$,

$$
t^{s}(M, p)=c^{s}(M, p)=a^{s}(M, p) .
$$

The inequalities $a^{s}(M, p) \leqslant c^{s}(M, p)$ and $a^{s}(M, p) \leqslant t^{s}(M, p)$ are proved in Lemma 2.19 and do not use the pseudoconvexity of $M$.

Thus, using the result of Lemma 2.19 to prove Theorem 2.14 one need only show that $c^{1}(M, p) \leqslant a^{1}(M, p)$.

2.17. REmarK. Example 5.9 shows that Theorem 2.14 is not valid, in general, if $M$ is not pseudoconvex. Also Example 5.9 or the examples of $[4, \S 3]$ provide simple examples of real hypersurfaces $M \subset C^{3}$ for which $t^{1}(M, p) \neq a^{1}(M, p)$. This situation contrasts with Theorem 2.12 where no assumption on the pseudoconvexity of $M$ is required. 
2.18. Remark. Let $M$ be a real-analytic pseudoconvex hypersurface in an open set $U \subset C^{n}$ and let $p \in M$. The work of J. J. Kohn [13] and Diederich and Fornaess [8] shows that there is a subelliptic estimate at $p$ in the $\bar{\partial}$-Neumann problem on forms of type $(r, s)$ if there are no germs of $s$-dimensional complex subvarieties $V$ with $p \in V \subset M$.

In view of Lemma 2.19 this theorem may be reformulated as follows: Let $M$ be a real analytic pseudoconvex hypersurface in an open set $U \subset \mathbf{C}^{n}$ and let $p \in M$. Then there is a subelliptic estimate at $p$ in the $\bar{\partial}$-Neumann problem for $(r, s)$ forms if for all $q$ near $p$ we have $c^{s}(M, p)<+\infty$, or for all $q$ near $p$ we have $t^{s}(M, p)<+\infty$.

This formulation is similar to a conjecture of J. J. Kohn [14]. (The conjecture as formulated by Kohn [14] is false.)

Combining Theorem 2.14 with a recent result of $\mathrm{D}$. Catlin [5] one obtains that if $p \in M \subset \mathbf{C}^{3}$ where $M$ is a $\bigodot^{\infty}$ pseudoconvex hypersurface, then an $\varepsilon$-subelliptic estimate does not hold at $p$ for $(0,1)$ forms if $\varepsilon>1 / c^{1}(M, p)$.

2.19. Lemma. Let $M$ be a real $\bigodot^{\infty}$ hypersurface of an open set $U \subset \mathbf{C}^{n}$ and let $p \in M$. For each integer $s, 1 \leqslant s \leqslant n-1, a^{s}(M, p)<t^{s}(M, p)$ and $a^{s}(M, p) \leqslant$ $c^{s}(M, p)$.

Proof. Let $X$ be an $s$-dimensional complex submanifold of a neighborhood of $p$ in $\mathbf{C}^{n}$ having contact of order $r$ with $M$ at $p$. It suffices to show that $t^{s}(M, p)>r$ and $c^{s}(M, p) \geqslant r$.

We may assume we have chosen local holomorphic coordinates $\left(z_{1}, \ldots, z_{n-1}, w\right)$ centered at $p$ so that $X=\left\{w=z_{s+1}=\cdots=z_{n-1}=0\right\}$ and the defining function $\rho$ for $M$ is in the form (1.2.4). Thus $\rho\left(z_{1}, \ldots, z_{s}, 0 \ldots 0\right)$ vanishes to order $\geqslant r$ at the origin.

Let $L_{1}, \ldots, L_{s}$ be the standard vector fields given by (1.4.2). Let $B$ be the subbundle of $T^{1,0}(M)$ which they span.

Now, if $g$ is a $\bigodot^{\infty}$ function such that $g \mid X$ vanishes to order $>l$ at $p$ then $L_{i}(g) \mid X$ vanishes to order $\geqslant \min (l-1, r-1)$ at $p$ (for $i=1, \ldots, s$ ); similarly for $\bar{L}_{i}(g)$. Also, the coefficient of $\partial / \partial w$ in $L_{i}$, namely $\partial \rho / \partial z_{i}$, has the property that $\left(\partial \rho / \partial z_{i}\right) \mid X$ vanishes to order $\geqslant r-1$ at $p$ (for $i=1, \ldots, s$ ); similarly for the coefficient of $\partial / \partial \bar{w}$ in $\overline{L_{i}}$, namely $\partial \rho / \partial \bar{z}_{i}$. Thus, if $F$ is an iterated commutator of $\mu$ of the vector fields $L_{1}, \ldots, L_{s}, \bar{L}_{1}, \ldots, \bar{L}_{s}$, we have

$$
F=\sum_{j=1}^{s} a_{j} \frac{\partial}{\partial z_{j}}+a_{n} \frac{\partial}{\partial w}+\sum_{j=1}^{s} b_{j} \frac{\partial}{\partial \bar{z}_{j}}+b_{n} \frac{\partial}{\partial \bar{w}}
$$

where $a_{n} \mid X$ and $b_{n} \mid X$ vanish to order $>r-\mu$ at $p$. We may conclude that $\langle F, \partial \rho\rangle(p)=0$ for all $F \in \mathfrak{R}_{r-1}(B)$ and hence $t(B, p) \geqslant r$. Thus $t^{s}(M, p)>r$.

With the same bundle $B$ as above, $\operatorname{tr}_{B} \mathcal{L}_{M}$, the trace of the Levi form of $M$ restricted to $B$, vanishes on $X$ to order $\geqslant r-2$. Thus, in a similar manner, we have $c^{s}(M, p) \geqslant r$. 


\section{Weighted coordinates.}

3.1. Definition. A weighted holomorphic coordinate system $(z ; \alpha)$ consists of the following.

(1) A local holomorphic coordinate system $\left(z_{1}, \ldots, z_{n}\right)$.

(2) Weights $\alpha_{1}, \ldots, \alpha_{n}$ assigned to the corresponding coordinates. Each $\alpha_{i}$ is an integer $\geqslant 1$ or $+\infty$.

For the problems considered in this paper the geometry of real submanifolds of $\mathbf{C}^{n}$ will be used to assign weights to the different directions in $\mathbf{C}^{n}$. For the use of weighted coordinates in various problems in analysis see [10] and [18].

3.2. Given a weighted holomorphic coordinate system $(z ; \alpha)$ centered at $p \in \mathbf{C}^{n}$ we assign a weight to any $e^{\infty}$ function $\phi$ defined in a neighbourhood of $p$ as follows. The function $\bar{z}_{i}$ is assigned the same weight as the coordinate function $z_{i}$, namely $\alpha_{i}$.

A monomial in $z, \bar{z}$ is assigned as weight the sum of the weights of all the $z_{i}$ and $\bar{z}_{i}$ occurring as a factor in it.

A $\bigodot^{\infty}$ function $\phi$ is assigned the weight $\gamma$ if, in the formal Taylor series expansion of $\phi$ at $p$, there is at least one monomial of weight $\gamma$ and all monomials are of weight $\geqslant \gamma$.

We use the notation $\operatorname{wt}(\phi)=\gamma$. Thus $\operatorname{wt}(\phi)=+\infty$ if $\phi$ is flat at $p$.

3.3. Immediate properties are $\left(\phi\right.$ and $\psi$ are $\bigcup^{\infty}$ functions defined in a neighbourhood of $p$ )

$$
\begin{aligned}
\operatorname{wt}(\phi \psi) & =\operatorname{wt}(\phi)+\operatorname{wt}(\psi) . \\
\operatorname{wt}(\phi+\psi) & \geqslant \min (\operatorname{wt}(\phi), \operatorname{wt}(\psi)) . \\
\operatorname{wt}\left(\partial \phi / \partial z_{i}\right) & \geqslant \operatorname{wt}(\phi)-\alpha_{i} . \\
\operatorname{wt}\left(\partial \phi / \partial \bar{z}_{i}\right) & \geqslant \operatorname{wt}(\phi)-\alpha_{i} . \\
\text { If } \operatorname{wt}(\phi) & >0 \text { then } \phi(p)=0 .
\end{aligned}
$$

3.4. Definition. A polynomial $\phi$ is homogeneous of weight $\mu$ if it is a sum of monomials each of which is of weight $\mu$.

3.5. We assign a weight to partial differential operators with $e^{\infty}$ coefficients defined in a neighbourhood of $p$ as follows (we do not assign a weight to operators involving differentiation in a coordinate assigned the weight $+\infty$. Thus the weight of a partial differential operator will be a positive or negative integer or $+\infty$ ).

$\partial / \partial z_{i}$ and $\partial / \partial \bar{z}_{i}$ are assigned the weight $-\alpha_{i}$.

An operator $(\partial / \partial z)^{I}(\partial / \partial \bar{z})^{J}$ is assigned as weight the sum of the weights $\partial / \partial z_{i}$ and $\partial / \partial \bar{z}_{i}$ occurring as a factor in it.

An operator

$$
Q=\sum_{I, J} \phi_{I J}\left(\frac{\partial}{\partial z}\right)^{I}\left(\frac{\partial}{\partial \bar{z}}\right)^{J}
$$

is assigned as weight

$$
\min _{I, J}\left\{w \mathrm{t}\left(\phi_{I J}\right)+\operatorname{wt}\left((\partial / \partial z)^{I}(\partial / \partial \bar{z})^{J}\right)\right\} .
$$

We use the notation $\operatorname{wt}(Q)$. 
We will denote by $\mathcal{S}$ the set of pairs of $n$-tuples for which the minimum in (3.5.2) is attained. That is

$$
\mathcal{S}=\left\{(I, J) \mid \mathrm{wt}(Q)=\operatorname{wt}\left(\phi_{I J}\right)+\operatorname{wt}\left((\partial / \partial z)^{I}(\partial / \partial \bar{z})^{J}\right)\right\} .
$$

3.6. Definition. A partial differential operator $Q$ is homogeneous if in the expression (3.5.1) the functions $\phi_{I J}$ are weighted homogeneous polynomials and each nonzero term $\phi_{I J}(\partial / \partial z)^{I}(\partial / \partial \bar{z})^{J}$ in (3.5.1) is of the same weight.

3.7. Immediate properties are $\left(Q_{1}\right.$ and $Q_{2}$ are partial differential operators with $e^{\infty}$ coefficients defined in a neighbourhood of $p$ )

$$
\operatorname{wt}\left(Q_{1} Q_{2}\right) \geqslant \operatorname{wt}\left(Q_{1}\right)+\operatorname{wt}\left(Q_{2}\right) .
$$

If $Q_{1}$ and $Q_{2}$ are homogeneous, then $Q_{1} Q_{2}$ is homogeneous and if $Q_{1} Q_{2} \not 0$ then $\operatorname{wt}\left(Q_{1} Q_{2}\right)=\operatorname{wt}\left(Q_{1}\right)+\operatorname{wt}\left(Q_{2}\right)$.

If $\phi$ is a $e^{\infty}$ function defined near $p \in \mathbf{C}^{n}$ then $\operatorname{wt}(Q(\phi))>$ $\operatorname{wt}(\phi)+\operatorname{wt}(Q)$. In particular, if $\operatorname{wt}(\phi)>|\mathbf{w t}(Q)|$ then $Q(\phi)(p)=0$.

3.8. For $\phi$ a $\bigodot^{\infty}$ function defined near $p$ we denote by $\phi^{\circ}$ the sum of the nonzero monomials in the Taylor series for $\phi$ at $p$ which are of lowest weight. $\phi^{\circ}$ is thus homogeneous of weight wt $(\phi)$.

Similarly, for $Q$ a partial differential operator given in the form (3.5.1) we denote by $Q^{\circ}$ the sum

$$
\sum_{I, J \in \delta} \phi_{I J}^{\circ}\left(\frac{\partial}{\partial z}\right)^{I}\left(\frac{\partial}{\partial \bar{z}}\right)^{J}
$$

\section{The homogeneous case.}

4.1. In this section we will solve (Theorem 4.2) a special case of Theorem 2.14. Then in $\$ 5$ we reduce the general case of a pseudoconvex hypersurface in $\mathbf{C}^{3}$ to this special case.

Theorem 4.2 states that for $M$ homogeneous in an appropriate sense (condition (a)) and pseudoconvex, if there is a tangential holomorphic vector field $L$ of a special form (condition (b)) with $c(L, p)=+\infty$ there is a 2-dimensional complex manifold contained in $M$ near $p$ (and hence $a^{2}(M, p)=+\infty$ ).

The conclusion of Theorem 4.2 is thus stronger than that of Theorem 2.14. This stronger conclusion is needed to carry out the process of reducing the general case to the special case of Theorem 4.2. It is the generalization of Theorem 4.2 which we have been unable to prove in $\mathbf{C}^{n}(n>3)$.

4.2. THEOREM. Let $M$ be a real $\bigodot^{\infty}$ hypersurface in $\mathbf{C}^{3}$ and let $p \in M$. Suppose that $M$ is pseudoconvex and that conditions (a) and (b) which follow are satisfied. Then there is a 2-dimensional complex manifold $X$ with $p \in X \subset M$.

(a) There is a weighted holomorphic coordinate system $\left(z_{1}, z_{2}, w\right)$ centered at $p$ with $\operatorname{wt}\left(z_{1}\right)=1, \operatorname{wt}\left(z_{2}\right)=k, \operatorname{wt}(w)=\mu$ where $1<k<\mu<+\infty$. The defining function $\rho$ for $M$ is in the form (1.2.4) and is weighted homogeneous. Thus we may write

$$
\rho=2 \operatorname{Re}(w)+h\left(z_{1}, \bar{z}_{1}, z_{2}, \bar{z}_{2}\right)
$$


where $h$ is a weighted homogeneous polynomial of weight $\mu$.

(b) There is a tangential holomorphic vector field $L$ such that

$$
\begin{gathered}
L(p) \neq 0, \\
c(L, p)=+\infty,
\end{gathered}
$$

$L=L_{1}+\phi L_{2}$ where $L_{1}$ and $L_{2}$ are the standard vector fields given by (1.4.2) and $\phi$ is a nonzero polynomial in $z_{1}, \bar{z}_{1}$, which is homogeneous of degree $k-1$ and not holomorphic.

4.3. Corollary. In (4.2.1) if the polynomial $h$ has no pure terms (that is, $h$ has no monomials in $z_{1}, z_{2}$ only or $\bar{z}_{1}, \bar{z}_{2}$ only) then $h \equiv 0$.

Proof (of Corollary 4.3 from Theorem 4.2). Let $u, v$ be holomorphic coordinates in a neighbourhood of $(0,0) \in \mathbf{C}^{2}$. Let $w(u, v), z_{1}(u, v), z_{2}(u, v)$ be a complex parametrization of $X$ near $p$. Since $C T(X, p) \simeq T^{1,0}(M, p) \oplus T^{0,1}(M, p)$ we may assume that $\left(\partial\left(z_{1}, z_{2}\right) / \partial(u, v)\right)(0,0) \neq 0$.

Substituting into the expression for $\rho$ we have

$$
\operatorname{Re}(w(u, v))=-h\left(z_{1}(u, v), \bar{z}_{1}(u, v), z_{2}(u, v), \bar{z}_{2}(u, v)\right) .
$$

The left-hand side of (4.3.1) involves only pure monomials while the right-hand side has no pure terms. Hence $h \equiv 0$.

4.4. The proof of Theorem 4.2 will be completed in $\$ 4.9$.

We will begin by considering the module $\mathfrak{T}$ (over the complex-valued real-analytic functions) generated by $L, \bar{L}$ and their commutators of all orders. The module is bracket closed and in the terminology of Freeman $[9, \S 3]$ it is a real module, i.e. $\mathfrak{N}=\overline{\mathfrak{K}}$. Then $\operatorname{Re}(\mathfrak{N})=\{\operatorname{Re}(D) \mid D \in \mathfrak{N}\}$ is bracket closed and by a theorem of Nagano [9] there is a unique integral manifold $Z$ passing through $p$. Thus $Z$ is a real-analytic submanifold of a neighbourhood of $p$ and for all $q \in Z$ near $p$

$$
T(Z, q)=\{\operatorname{Re}(D)(q) \mid D \in \mathfrak{N}\}
$$

and

$$
\mathrm{CT}(Z, q) \simeq\{D(q) \mid D \in \mathscr{N}\}
$$

It follows that

$$
\operatorname{dim}_{\mathbf{R}} Z=\operatorname{dim}_{\mathbf{R}}\{\operatorname{Re}(D(p)) \mid D \in \mathfrak{T}\}=\operatorname{dim}_{\mathbf{C}}\{D(p) \mid D \in \mathfrak{N}\} .
$$

It is clear from the characterization of $Z$ given by Freeman [9, Theorem 2.1] that since $L$ and $\bar{L}$ are weighted homogeneous of weight -1 then $Z$ is also weighted homogeneous. That is $Z$ is defined by the vanishing of weighted homogeneous polynomials. $Z$ will thus be, in fact, an algebraic submanifold of $\mathbf{C}^{n}$.

4.5. Now we use the assumption that $c(L, p)=+\infty$. Thus, for all sets of positive integers $k, i_{0}, \ldots, i_{k}, j_{0}, \ldots, j_{k}$ we have

$$
L^{i_{0}} \bar{L}^{j_{0}} \cdots L^{i_{k}} \bar{L}^{j_{k}}\langle[L, \bar{L}], \partial \rho\rangle(p)=0 .
$$

We conclude that $\langle[L, \bar{L}], \partial \rho\rangle$ vanishes on $Z$. Alternatively, $\mathcal{L}_{M}(q, L(q))=0$ for all $q \in Z$. 
Let $A=\operatorname{Re}(L)$ and $B=\operatorname{Im}(L)$. Then, using the notation of 1.5 we have $A$, $B \in T^{N}(Z)$. We also note that the module over the real-valued real-analytic functions generated by $A$ and $B$ together with their commutators of all lengths is $\operatorname{Re}(\mathfrak{R})$.

4.6. LEMMA. $\operatorname{dim}_{\mathbf{R}}(Z)=3$ or 4 .

Proof. Since $A$ and $B \in T^{N}(Z)$ and $M$ is pseudoconvex, all commutators of $A$ and $B$ are in $T^{N}(Z)$ [8, Proposition 2]. In particular, these commutators are all in $T^{h}(M)$. That is $\{\operatorname{Re} D(p) \mid D \in \mathfrak{N}\} \subset T^{h}(M, p)$. Since $\operatorname{dim}_{\mathrm{R}} T^{h}(M, p)=4$ we may conclude that $\operatorname{dim}_{\mathbf{R}} Z \leqslant 4$.

To show that $\operatorname{dim}_{\mathbf{R}} Z \geqslant 3$ we will show that $\operatorname{dim}_{\mathbf{C}}\{D(p) \mid D \in \Re\} \geqslant 3$. To this end we introduce the operator

$$
S=\partial / \partial z_{1}+\phi\left(\partial / \partial z_{2}\right) \quad \text { where } \phi \text { is given by (4.2.4). }
$$

It follows from (1.4.2) that

$$
L=S-S(\rho)(\partial / \partial w)
$$

It is clear that $\partial / \partial z_{1}$ and $\partial / \partial \bar{z}_{1} \in\{D(p) \mid D \in \mathscr{R}\}$. We must show there is an iterated commutator of $L, \bar{L}$ whose value at $p$ is not in the span of $\partial / \partial z_{1}$ and $\partial / \partial \bar{z}_{1}$. This will be done in Lemma 4.8.

4.7. Let $C$ be an iterated commutator in the noncommuting indeterminates $X, Y$. We denote by $C_{1}$ the sum of those monomials in the expansion of $C(X, Y)$ with $X$ on the right and by $C_{2}$ the sum of those monomials with $Y$ on the right.

For $Q_{1}$ and $Q_{2}$ partial differential operators we denote by $C\left(Q_{1}, Q_{2}\right)$ the partial differential operator obtained by substituting $Q_{1}$ and $Q_{2}$ for $X$ and $Y$ respectively in $C(X, Y)$; similarly for $C_{1}$ and $C_{2}$.

For $L$ and $S$ the vector fields given by (4.2.4) and (4.6.2) it follows from [2, Lemma 5.11] that

$$
C(L, \bar{L})=C(S, \bar{S})-C_{1}(S, \bar{S})(\rho)(\partial / \partial w)-C_{2}(S, \bar{S})(\rho)(\partial / \partial \bar{w}) .
$$

4.8. LEMMA. There is a commutator $C$ of length $k$ in $S, \bar{S}$, such that $C(S, \bar{S})$ is not in the span of $\partial / \partial z_{1}$ and $\partial / \partial \bar{z}_{1}$.

Proof. Direct computation shows that

$$
[S, \bar{S}]=\left(\partial \bar{\phi} / \partial z_{1}\right)\left(\partial / \partial \bar{z}_{2}\right)-\left(\partial \phi / \partial \bar{z}_{1}\right)\left(\partial / \partial z_{2}\right)
$$

For $k=2$ the proof is now complete since, in this case, $\phi$ is of the form $\alpha \bar{z}_{1}+\beta z_{1}$ with $\alpha \neq 0$, and hence the coefficients of $\partial / \partial z_{2}$ and $\partial / \partial \bar{z}_{2}$ in (4.8.1) are nonzero.

For $k>2$ we first note that since $\phi$ depends only on $z_{1}, \bar{z}_{1}$ then $[S, \bar{S}]$ commutes with $\phi\left(\partial / \partial z_{2}\right)$. Thus if we consider an iterated commutator of the form $C(S, \bar{S})=$ $[\ldots[S, \bar{S}]]$ involving $\gamma$ of $S$ and $\delta$ of $\bar{S}(\gamma+\delta=k, \gamma>1, \delta>1)$ we have

$$
C(S, \bar{S})=\frac{\partial \bar{\phi}}{\partial z_{1}^{\gamma} \partial \bar{z}_{1}^{\delta-1}} \frac{\partial}{\partial \bar{z}_{2}}-\frac{\partial \phi}{\partial z_{1}^{\gamma-1} \partial \bar{z}_{1}^{\delta}} \frac{\partial}{\partial z_{2}} .
$$

Since $\phi$ is homogeneous of degree $k-1$ and not holomorphic one may choose $\gamma$ and $\delta$ so that the coefficient of $\partial / \partial z_{2}$, or that of $\partial / \partial \bar{z}_{2}$, is nonzero. 
The proof of Lemma 4.8 is now complete. We note that Lemma 4.8 together with (4.7.1) completes the proof of Lemma 4.6.

4.9. We will now complete the proof of Theorem 4.2. First we consider the case that $\operatorname{dim}_{\mathrm{R}} Z=4$. Since $A, B$ and their commutators of all lengths have values in $T^{h}(M, q)$ for $q \in Z$ (see 4.5) we may conclude that $L, \bar{L}$ and their commutators of all lengths span $T^{1,0}(M, q) \oplus T^{0,1}(M, q)$ for $q \in Z$. Thus $\operatorname{CT}(Z, q)$ is invariant under the almost complex structure of $\mathbf{C}^{3}$ and we may conclude that $Z$ is a complex submanifold.

Next we must consider the case that $\operatorname{dim}_{\mathrm{R}} Z=3$. In this case $T^{1,0}(Z, q)$ is of constant dimension one, being spanned at each point $q \in Z$ by $L(q)$. $Z$ is thus a $C-R$ submanifold.

By a result of Rossi [16] (see also [8, Proposition 1]) we may choose new local holomorphic coordinates $\left(z_{1}, z_{2}, w\right)$ centered at $p$ such that $Z \subset\{w=0\}$.

Now conditions (a), (b) and (c) of Proposition 3 of Diederich and Fornaess [8] are satisfied. We may conclude that in a neighbourhood of $p,\left\{\left(z_{1}, z_{2}, w\right) \mid w=0\right\} \subset$ $M$.

\section{Reduction to the homogeneous case.}

5.1. In this chapter we will reduce the general case of Theorem 2.14 to the special case of Theorem 4.2 .

There are a number of special cases in which Theorem 2.14 holds without the pseudoconvexity assumption on $M$ near $p \in M$. Some of these are dealt with in Remark 5.3 and 5.4. Once these cases are eliminated and correct local holomorphic coordinates are chosen the reduction to the homogeneous case (i.e., Theorem 4.2) is carried out in 5.7 and 5.8 .

In 5.9 we give an example to show that Theorem 2.14 (or Theorem 4.2) is not valid, in general, without a pseudoconvexity assumption on $\boldsymbol{M}$.

First, we need a lemma which will give the effect of certain holomorphic changes of coordinates on the standard vector fields $L_{i}$ (see (1.4.2)).

5.2. Lemma. Let $M$ be a real $e^{\infty}$ hypersurface in an open set $U \subset \mathbf{C}^{n}$ and $p \in M$ the origin of coordinates. Let $\rho$ be a defining function for $M$ in the form (1.2.4).

(1) Let $w, z_{1}^{\prime}, \ldots, z_{n-1}^{\prime}$ be new local holomorphic coordinates where $z_{j}^{\prime}=$ $z_{j}\left(z_{1}, \ldots, z_{n-1}\right)$ for $j=1, \ldots, n-1$. Then

$$
L_{i}^{\prime}=\sum_{j=1}^{n-1} \frac{\partial z_{j}}{\partial z_{i}^{\prime}} L_{j} \quad \text { for } i=1, \ldots, n-1
$$

(2) Let $w^{\prime}=w^{\prime}\left(w, z_{1}, \ldots, z_{n-1}\right)$ be a new complex normal coordinate with $z_{1}, \ldots, z_{n-1}$, unchanged. Then

$$
L_{i}^{\prime}=\left(\partial w / \partial w^{\prime}\right) L_{i} \text { for } i=1, \ldots, n-1 .
$$

Here $L_{i}^{\prime}$ denotes the standard vector field with respect to the new coordinates. We assume that in the new coordinates $\rho$ again has the form (1.2.4). 
Proof. Chain rule.

5.3. REMARK. If $a^{1}(M, p)=+\infty$ then Theorem 2.14 follows from Lemma 2.19 (without any pseudoconvexity assumption on $M$ in the statement of Theorem 2.14).

5.4. We now restrict ourselves to the case $M \subset C^{3}$. Let $\rho\left(z_{1}, z_{2}, w\right)$ be a defining function for $M$ near $p \in M$ in the form (1.2.4). Suppose that $\rho\left(z_{1}, 0,0\right)$ vanishes at $z_{1}=0$ to order $s$ and let $Q\left(z_{1}\right)$ denote the terms of order $s$ in the Taylor series for $\rho\left(z_{1}, 0,0\right)$ at $z_{1}=0$. If $Q\left(z_{1}\right)$ is harmonic, say $Q\left(z_{1}\right)=\operatorname{Re}\left(H\left(z_{1}\right)\right)$ where $H\left(z_{1}\right)$ is analytic, we may introduce a new complex normal coordinate

$$
w^{\prime}=w-H\left(z_{1}\right) \text {. }
$$

Then, with respect to the new coordinates $\left(z_{1}, z_{2}, w^{\prime}\right), \rho\left(z_{1}, 0,0\right)$ vanishes at $z_{1}=0$ to order $>s$. The above procedure may be repeated if the lowest-order terms in the Taylor series of $\rho\left(z_{1}, 0,0\right)$ at $z_{1}=0$ are again harmonic.

If the above procedure does not stop, that is, if at all stages the lowest-order terms in $\rho\left(z_{1}, 0,0\right)$ are harmonic then $a^{1}(M, p)=+\infty$.

5.5. LEMMA. Let $\rho$ be a defining function near $p \in M$ for $M \subset \mathbf{C}^{3}$ and suppose $\rho\left(z_{1}, 0,0\right)$ vanishes at $z_{1}=0$ to order $s$ and $Q\left(z_{1}\right)$ is not harmonic. Let $L=L_{1}+$ $\phi L_{2}$ be a tangential holomorphic vector field where $L_{1}, L_{2}$ are the standard vector fields, $\phi$ is $e^{\infty}$ and $\phi\left(z_{1}, 0,0\right)$ vanishes to order $k-1$ at $z_{1}=0$. Assume $k>2$. Then $s<k$ implies that $c(L, p) \leqslant s$.

Proof. For some integers $\alpha, \beta$ with $\alpha+\beta=s, \alpha \geqslant 1, \beta \geqslant 1$, we have

$$
\left(\partial / \partial z_{1}\right)^{\alpha}\left(\partial / \partial \bar{z}_{1}\right)^{\beta} \rho\left(z_{1}, 0,0\right) \neq 0 .
$$

Consider $L^{\alpha-1}(\bar{L})^{\beta-1}\langle[L, \bar{L}], \partial \rho\rangle(p)$. We will show this is nonzero. First we note that if $g$ is $e^{\infty}$ near $p$ and $g\left(z_{1}, 0,0\right)$ vanishes at $z_{1}=0$ to order $l$ then $L_{1}(g)\left(z_{1}, 0,0\right)$ vanishes at $z_{1}=0$ to order $\geqslant \min (l-1, s-1)$ (similarly for $\left.\bar{L}_{1}(g)\left(z_{1}, 0,0\right)\right)$. Hence if $L^{\alpha-1}(\bar{L})^{\beta-1}=\left(L_{1}+\phi L_{2}\right)^{\alpha-1}\left(\bar{L}_{1}+\bar{\phi} \bar{L}_{2}\right)^{\beta-1}$ is expanded as a sum of monomials in $L_{1}, \bar{L}_{1}, L_{2}, \bar{L}_{2}$ the only term with a coefficient which is nonzero at $p$ is $L_{1}^{\alpha-1} \bar{L}_{1}^{\beta-1}$. Also $\langle[L, \bar{L}], \partial \rho\rangle=\left\langle\left[L_{1}, \bar{L}_{1}\right], \partial \rho\right\rangle+h$ where $h$ is $e^{\infty}$ near $p$ and $h\left(z_{1}, 0,0\right)$ vanishes to order $\geqslant k-2 \geqslant s-1$ at $z_{1}=0$.

Thus, combining the above with (5.5.1) and the result [3, Theorem 3] that

$$
L_{1}^{\alpha-1}\left(\bar{L}_{1}\right)^{\beta-1}\left\langle\left[L_{1}, \bar{L}_{1}\right], \partial \rho\right\rangle(p)=\left(\partial / \partial z_{1}\right)^{\alpha}\left(\partial / \partial \bar{z}_{1}\right)^{\beta} \rho\left(z_{1}, 0,0\right),
$$

we conclude that $c(L, p) \leqslant s$.

5.6. Let $L=L_{1}+\phi L_{2}$ be a tangential holomorphic vector field with $\phi\left(z_{1}, 0,0\right)$ vanishing at $z_{1}=0$ to order $k-1$. Suppose that the terms of order $k-1$ in the Taylor series of $\phi\left(z_{1}, 0,0\right)$ at $z_{1}=0$ are analytic. That is, they are of the form $c z_{1}^{k-1}$ with $c \neq 0$. We then introduce a new $z_{1}$ coordinate

$$
z_{1}^{\prime}=z_{1}+c z_{1}^{k-1} z_{2}
$$

Applying Lemma 5.2 we have

$$
u\left(L_{1}+\phi L_{2}\right)=L_{1}^{\prime}-c z_{1}^{k-1} L_{2}^{\prime}+u c z_{1}^{k-1} L_{2}^{\prime}
$$

where $u$ is a $\bigodot^{\infty}$ function with $u(p)=1$. Thus, the vector field $L^{\prime}=L_{1}^{\prime}+\psi L_{2}^{\prime}$ for $\psi=c z_{1}^{k-1}(u-1)$ has the properties 
(1) $c\left(L^{\prime}, p\right)=c(L, p)$,

(2) $\psi\left(z_{1}^{\prime}, 0,0\right)$ vanishes at $z_{1}^{\prime}=0$ to order $\geqslant k$.

We also note that under the change of coordinate (5.6.1) $\left\{\left(z_{1}, z_{2}, w\right) \mid z_{2}=w=0\right\}$ remains fixed. Thus, for example the function $\rho\left(z_{1}, 0,0\right)$ remains fixed.

5.7. Let $M$ be a real $e^{\infty}$ hypersurface in $\mathbf{C}^{3}$ and $p \in M$. Let $\rho$ be a defining function for $M$ near $p$.

To prove Theorem 2.14 we must show that if $M$ is pseudoconvex near $p$ then $c^{1}(M, p) \leqslant a^{1}(M, p)$ and we may assume that $a^{1}(M, p)<+\infty$.

Thus, let $L$ be a tangential holomorphic vector field with $L(p) \neq 0$ and assume $c(L, p)>a^{1}(M, p)$. We will proceed by contradiction. The contradiction will be obtained in 5.8 based on Theorem 4.2.

Assume the $z_{1}, z_{2}$ coordinates are chosen so that $L(p)=L_{1}(p)$. We first proceed as in 5.4 and change the complex normal coordinate so that the lowest-order terms in the Taylor series of $\rho\left(z_{1}, 0,0\right)$ at $z_{1}=0$ are not harmonic. Suppose that after this change, $\rho\left(z_{1}, 0,0\right)$ vanishes to order $s$ at $z_{1}=0$. We then do one more change in the complex normal coordinate of the form (5.4.1) to remove any pure monomials in the lowest order terms in the Taylor series of $\rho\left(z_{1}, 0,0\right)$ at $z_{1}=0$.

After multiplying $L$ by a nowhere zero $e^{\infty}$ function we may assume it is in the form

$$
L=L_{1}+\phi L_{2} \quad \text { where } \phi(0)=0 .
$$

Then we use the procedure of 5.6 to change the $z_{1}$-coordinate in the case that the lowest-order term in the Taylor series of $\phi\left(z_{1}, 0,0\right)$ at $z_{1}=0$ is analytic. We may still assume $L$ is in the form (5.7.1) and that $\rho\left(z_{1}, 0,0\right)$ is unaffected by these changes.

Suppose that $\phi\left(z_{1}, 0,0\right)$ vanishes to order $k-1$ at $z_{1}=0$. Then, since $c(L, p)>$ $a^{1}(M, p) \geqslant s$ we must have, by Lemma 5.5 , that $k \leqslant s$. In particular, since the procedure of 5.6 increases the order of vanishing of $\phi\left(z_{1}, 0,0\right)$ at $z_{1}=0$ by at least one, the procedure must stop after a finite number of steps. We thus may assume that the lowest-order terms in the Taylor series of $\phi\left(z_{1}, 0,0\right)$ at $z_{1}=0$ are not analytic. We still denote its order of vanishing by $k-1$.

We now introduce weights for the local holomorphic coordinate system. We set $\mathrm{wt}\left(z_{1}\right)=1, \mathrm{wt}\left(z_{2}\right)=k$. We then introduce a new complex normal coordinate

$$
w^{\prime}=w-F\left(z_{1}, z_{2}\right)
$$

where $\operatorname{Re}\left(F\left(z_{1}, z_{2}\right)\right)$ denotes the sum of the pure monomials of lowest weight in the Taylor series of $\rho\left(z_{1}, z_{2}, 0\right)$ at the origin.

Since the terms of lowest order in the Taylor series of $\rho\left(z_{1}, 0,0\right)$ at $z_{1}=0$ contain no pure monomials we have $F\left(z_{1}, 0\right) \equiv 0$ since $F$ can have no monomials in $z_{1}$ or $\bar{z}_{1}$ only. Thus the change of coordinate (5.7.2) leaves the $z_{1}$-axis, $\phi\left(z_{1}, 0,0\right)$, $\rho\left(z_{1}, 0,0\right)$, and the integers $s$ and $k$ fixed. We repeat the change of coordinate (5.7.2) as often as possible. Then, we assign to the final complex normal coordinate $w$ the weight

$$
w t(w)=w t\left(\rho\left(z_{1}, z_{2}, 0\right)\right)
$$

which we will denote by $\mu$. 
5.8. Thus we may assume we are in the following situation. We have local analytic coordinates $\left(z_{1}, z_{2}, w\right)$ with weights $\operatorname{wt}\left(z_{1}\right)=1, \operatorname{wt}\left(z_{2}\right)=k>1$ and $\operatorname{wt}(w)$ $=\mu$. The defining function is in the form (1.2.4) and $\operatorname{wt}(\rho)=\mu=\operatorname{wt}\left(\rho\left(z_{1}, z_{2}, 0\right)\right)$. There are no pure terms in $\left(\rho\left(z_{1}, z_{2}, 0\right)\right)$ so we have $\mu \geqslant k$.

There is a tangential holomorphic vector field $L=L_{1}+\phi L_{2}$ where $\phi\left(z_{1}, 0,0\right)$ vanishes at $z_{1}=0$ to order $k-1 . \phi^{\circ}$ is not analytic. $c(L, p)=m>s>\mu$. We will derive a contradiction by showing that $m>\mu$ is impossible.

Now $w t(L)=-1$. Then (see 3.8)

$$
L^{\circ}=L_{1}^{\circ}+\phi^{\circ} L_{2}^{\circ}
$$

where

$$
L_{i}^{\circ}=\left(\partial \rho^{\circ} / \partial z_{1}\right)(\partial / \partial w)-\left(\partial \rho^{\circ} / \partial w\right)\left(\partial / \partial z_{i}\right) \text { for } i=1,2 .
$$

We note that $\operatorname{wt}\left(L-L^{\circ}\right) \geqslant 0$.

We will show that $\mu<m$ implies that $\left(\rho\left(z_{1}, z_{2}, 0\right)\right)^{\circ} \equiv 0$ and this contradiction will establish the result.

$\rho^{\circ}=2 \operatorname{Re}(w)+\left(\rho\left(z_{1}, z_{2}, 0\right)\right)^{\circ}$ and $L^{\circ}$ is a tangential holomorphic vector field to the manifold $M^{\circ}=\left\{\rho^{\circ}=0\right\}$. Furthermore $M^{\circ}$ is pseudoconvex if $M$ is [3, $\left.\S 2\right]$.

$\left\langle\left[L^{\circ}, \bar{L}^{\circ}\right], \partial \rho^{\circ}\right\rangle$ is either $\equiv 0$ or homogeneous of weight $\mu-2$. Thus, since $L^{\circ}$ is homogeneous of weight -1 either $c\left(L^{\circ}, p\right)=\mu$ or $c\left(L^{\circ}, p\right)=+\infty$. However, by Corollary 4.3, $c\left(L^{\circ}, p\right)=+\infty$ implies that $\left(\rho\left(z_{1}, z_{2}, 0\right)\right)^{\circ} \equiv 0$. Hence we need only show that $c\left(L^{\circ}, p\right)=+\infty$.

If $\left\langle\left[L^{\circ}, \bar{L}^{\circ}\right], \partial \rho^{\circ}\right\rangle$ is not $\not \neq 0$ then $\langle[L, \bar{L}], \partial \rho\rangle^{\circ}=\left\langle\left[L^{\circ}, \bar{L}^{\circ}\right], \partial \rho^{\circ}\right\rangle$ and for any sets of integers $n, i_{0}, \ldots, i_{n}, j_{0}, \ldots, j_{n}$ with $\sum_{t=0}^{n} i_{t}+j_{t}=\mu-2$ we have

$$
\begin{aligned}
L^{i_{0}} \bar{L}^{j_{0}} \ldots L^{i_{n}} \bar{L}^{j_{n}}\langle[L, \bar{L}], \partial \rho\rangle(p) & \\
& =\left(L^{\circ}\right)^{i_{0}}\left(L^{\circ}\right)^{j_{0}} \ldots\left(L^{\circ}\right)^{i_{n}}\left(\overline{L^{\circ}}\right)^{j_{n}}\left\langle\left[L^{\circ}, \bar{L}^{\circ}\right], \partial \rho^{\circ}\right\rangle(p) .
\end{aligned}
$$

Thus if $c(L, p)=m>\mu$ then $c\left(L^{\circ}, p\right)=+\infty$.

5.9. Example. Let $\rho=2 \operatorname{Re}(w)+\left(z_{2}+\bar{z}_{2}+\left|z_{1}\right|^{2}\right)^{2}$ and let $M=\left\{\left(z_{1}, z_{2} ; w\right) \in\right.$ $\left.C^{3} \mid \rho=0\right\}$. Let $p=(0,0,0)$. Then we will show that $a^{1}(M, p)=4$ but $c^{1}(M, p)=$ $t^{1}(M, p)=+\infty$.

$M$ is not pseudoconvex near $p$ and this example shows that Theorem 2.14 is not valid, in general, if $M$ is not pseudoconvex.

It is easy to check that $a^{1}(M, p)=4$ and the maximum order of contact is realized by, for example, the $z_{1}$-axis.

Let $L=L_{1}-\bar{z}_{1} L_{2}$. Then (see (4.6.1) and (4.6.2)) $L=S-S(\rho)(\partial / \partial w)$ where $S=\partial / \partial z_{1}-\bar{z}_{1}\left(\partial / \partial z_{2}\right)$. However, direct computation shows that, in this example, $S(\rho) \equiv 0$ so that $L=S$. Now

$$
\langle[L, \bar{L}], \partial \rho\rangle=2\left(z_{2}+\bar{z}_{2}+\left|z_{1}\right|^{2}\right) \text { and } L\left(z_{2}+\bar{z}_{2}+\left|z_{1}^{2}\right|\right) \equiv 0 .
$$

Thus $c(L, p)=+\infty$ and so $c^{1}(M, p)=+\infty$.

Since all commutators of length $>3$ in $L, \bar{L}$ are $\equiv 0$ we may conclude that $t^{\prime}(M, p)=+\infty$.

A related set of examples may be found in [4, §3]. 
We also note that with $\operatorname{wt}\left(z_{1}\right)=1, \operatorname{wt}\left(z_{2}\right)=2$ and $\operatorname{wt}(w)=4, M$ satisfies the conditions of Theorem 4.2 except that it is not pseudoconvex. Thus pseudoconvexity is necessary, in general, in Theorem 4.2 also.

\section{REFERENCES}

1. T. Bloom and I. Graham, A geometric characterization of points of type $m$ on real submanifolds of $C^{n}$, J. Differential Geometry 12 (1977), 171-182.

2. ___ On 'type' conditions for generic real submanifolds of $\mathbf{C}^{\mathbf{n}}$, Invent. Math. 40 (1977), 217-243.

3. T. Bloom, Remarks on type conditions for real hypersurfaces in $\mathbf{C}^{n}$ (Proc. Internat. Conf. on Several Complex Variables, Cortona, Italy, 1976-77), Scuola Norm. Sup. Pisa, Pisa, 1978, pp. 14-24.

4. __ Sur le contact entre sous-variétés réelles et sous-variétés complexes de $\mathbf{C}^{n}$, Séminaire Pierre Lelong, 1975/76, Lecture Notes in Math., no. 578, Springer-Verlag, Berlin and New York, 1977, pp. $28-43$.

5. D. Catlin, Necessary conditions for subellipticity and hypoellipticity for the $\bar{\partial}$-Neumann problem on pseudoconvex domains (Proc. Conf. Several Complex Variables, Princeton, N. J., 1979) (to appear).

6. J. D'Angelo, Finite type conditions for real hypersurfaces, J. Differential Geometry (to appear).

7. M. Derridj, Estimations pour $\bar{\partial}$ dans des domaines non pseudo-convexes, Ann. Inst. Fourier (Grenoble) 28 (1978), 239-254.

8. K. Diederich and J.-E. Fornaess, Pseudoconvex domains with real analytic boundary, Ann. of Math. (2) 107 (1978), 371-384.

9. M. Freeman, Integration of analytic differential systems with singularities and some applications to real submanifolds of $\mathbf{C}^{n}$, J. Math. Soc. Japan 30 (1978), 571-578.

10. R. Goodman, Nilpotent Lie groups: structure and applications to analysis, Lecture Notes in Math., no. 562, Springer-Verlag, Berlin and New York, 1976.

11. P. C. Greiner, On subelliptic estimates for the $\bar{\partial}-$ Neumann problem in $\mathbf{C}^{2}$, J. Differential Geometry 9 (1974), 239-250.

12. J. J. Kohn, Boundary behaviour of $\bar{\partial}$ on weakly pseudo-convex manifolds of dimension two, J. Differential Geometry 6 (1972), 523-542.

13. , Subellipticity of the $\bar{\partial}-$ Neumann problem on pseudo-convex domains: sufficient conditions, Acta Math. 142 (1979), 79-122.

14. Subellipticity of the $\bar{\partial}$-Neumann problem on weakly pseudo-convex domains, Rencontres sur l'Analyse Complexe à Plusieurs Variables et les Systèmes Indéterminés, Université de Montréal Press, Montréal, 1975, pp. 105-118.

15. Sufficient conditions for subellipticity on weakly pseudo-convex domains, Proc. Nat. Acad. Sci. U.S.A. 74 (1977), 2214-2216.

16. S. G. Krantz, Characterizations of various domains of holomorphy via $\bar{\partial}$ estimates and applications to a problem of Kohn, Illinois J. Math. 23 (1979), 267-285.

17. H. Rossi, Differentiable manifolds in complex Euclidean space (Proc. Internat. Congr. Math., Moscow, 1966), “Mir”, Moscow, 1968, pp. 512-516.

18. L. Rothschild and E. M. Stein, Hypoelliptic differential operators and nilpotent groups, Acta Math. 137 (1976), 247-320.

Department of Mathematics, University of Toronto, Toronto, Ontario, Canada m5S 1al 